\title{
Asociación entre actividad física, estado de salud y patología de hombro en adultos chilenos Relationship between physical activity, health status, and shoulder pathology in adults from Chile Félix Zurita-Ortega, Ramón Chacón Cuberos, Manuel Fernández-Sánchez, Manuel Castro-Sánchez Universidad de Granada (España)
}

Resumen. Este estudio de carácter descriptivo y de corte transversal se realizó sobre un grupo de 41 adultos mayores de Santiago de Chile (Chile) que sufrían patología de hombro, con una edad media de 58,17 años $(D T=11,75)$. Se utilizó para la valoración el Test UCLA, el Termómetro de EUROQOL de Autovaloración del Estado de Salud y un Cuestionario Sociodemográfico. El análisis de datos se realizó a través de SPSS 20.0 empleando descriptivos básicos y un modelo de regresión logística binaria. Los sujetos que presentaron tendinopatías en el hombro fueron un 53,7\% (n = 22). Asimismo, un 48,8\% indicó que practicaba actividad física de forma continuada, únicamente un 14,6\% (n=6) no lo hacía periódicamente y un 39\% (n = 16) desarrollaba ejercicios de mantenimiento. El estado de salud y la evaluación UCLA mostraron asociaciones estadísticas. El modelo de regresión reveló asociaciones de la actividad física con el sexo [OR =0,086 (0,008-0,977)] y el estado de salud [OR = 0,021 (0,001-0,311)]. Como principales conclusiones se concreta que la actividad física regular es un elemento que disminuye el desarrollo de dolor en la patología de hombro en mayores. Asimismo, los datos obtenidos revelan que ser mujer y tener peor estado de salud se asocia a una menor práctica de actividad física en personas con patología de hombro.

Palabras Clave: Hombro, Salud, Actividad Física, Adulto Mayor.

\begin{abstract}
This descriptive and cross-sectional study was performed on a group of 41 adults from Santiago of Chile (Chile) who suffer shoulder pathology, with an average age of 58,17 years old $(S D=11,75)$. This study employed as main instruments the EUROQOL THERMOMETER, the UCLA scale, a self-assessment for health status, and a sociodemographic questionnaire. This study used SPSS 20.0 software for data analysis employing basic descriptive analysis and a f binary logistic regression model. A total of 53,7\% $(n=22)$ of the subjects showed shoulder tendinopathies. The $48,8 \%$ of the sample reported that they do physical activity frequently, while only $14,6 \%(n=6)$ indicated that they never do physical activity regularly, and $39 \%(n=16)$ do maintenance exercise. The regression model showed associations between physical activity and sex [OR = 0,086 $(0,008-0,977)]$ and health status [OR $=0,021(0,001$ to 0,311$)$. As main conclusions, regular physical activity is an element that decreases the development of pain in shoulder pathology. Likewise, the data obtained reveal that being a woman and having a worse state of health is associated with less physical activity in people affected by shoulder pathology.
\end{abstract}

Key words: Shoulder, Health, Physical Activity, Elderly.

\section{Introducción}

El estudio del hombro y las incapacidades que ocasiona su alteración es un tema actual en la población adulta mayor, con especial énfasis en el contexto sanitario y educacional (Hegedus et al., 2015). Diversos estudios muestran la importancia de prevenir y actuar interventivamente ante la aparición de diversas patologías en edades adultas y concretamente las que afectan al síndrome subacromial, que se refiere fundamentalmente al cuadro de dolor localizado en la región anterior del hombro y que está en estrecha relación con los movimientos combinados de flexión - abducción (Razmjou et al., 2015; Sharma, Bærheim, \& Kvåle, 2015).

La literatura asociada a este tipo de problemática la aborda vinculada a múltiples sinónimos, como el síndrome de hombro doloroso, síndrome del arco subacromial, pinzamiento del manguito rotador, bursitis subacromial, tendinosis del manguito rotador o tendinopatía del supraespinoso (Calvo, Arce, \& Calvo, 2009), cimentando la premisa de que el hombro es la articulación más inestable del cuerpo humano y que los procesos de envejecimiento están vinculados a un deterioro óseoarticular que se hace patente en esta articulación (Breckenridge \& McAuley, 2011; Patel, 2009).

La detección precoz de patologías de hombro resulta esencial en su tratamiento con el fin de mejorar la calidad de vida y el estado de salud percibida de este colectivo. Por este motivo, se concretan diversas técnicas de diagnóstico con el fin de evitar cirugía y mayores periodos incapacidad y rehabilitación (Parra et al., 2016). Sin embargo, la utilización de técnicas no invasivas son objeto de debate en numerosos contextos (Hinds et al., 2015; Waterman et al., 2015), obviando los beneficios asociados a este tipo de tratamientos y la ejecución de un diagnóstico temprano y el tratamiento precoz de las deformidades de hombro, lo cual evitaría intervenciones quirúrgicas futuras.

Una de las principales causas que se relacionan con la aparición de patología de hombro es la realización de actividad física cotidiana que implica una elevación del brazo por encima de la cabeza o flexiones

Fecha recepción: 18-04-18. Fecha de aceptación: 26-09-18 Ramón Chacón Cuberos

rchacon@ugr.es superiores a $180^{\circ}$, lo cual suele estar vinculado a ciertos colectivos profesionales o especialidades deportivas concretas (Calvo et al., 2009; Karel, 2017). De forma más específica, las lesiones del manguito rotador son las más frecuentes en grupos de avanzada edad que por su actividad profesional adoptan posturas mantenidas en abducción del hombro o levantan pesos por encima de la cabeza con escasas nociones de higiene postural (Jimenez-Olmedo, Penichet-Tomás, Pueo, Chinchilla-Mira, \& Pérez-Turpín, 2018; Kappe, Knappe, Elsharkawi, Reichel, \& Cakir, 2015).

En las últimas décadas el estudio de las lesiones de hombro asociadas a la práctica de actividad deportiva ha sido abundante (Crotin, Bhan, \& Ramsey, 2015)y más concretamente de los efectos de la lesión en el deporte de alta competición (Peixoto et al., 2013). Sin embargo, los estudios realizados en la población adulta vinculada a otras prioridades de tipo familiar o laboral son escasos o se limitan principalmente al diagnóstico y tratamiento rehabilitador (Breckenridge \& McAuley, 2011; Furness, Schram, Cottman-Fields, Solia, \& Secomb, 2018; Sharma et al., 2015). Por ello, resulta de interés conocer los tipos de patologías de hombro más frecuentes y cuál es su relación con el estado de salud percibido y su nivel de práctica de actividad física dado que esta puede actuar como factor de riesgo y/o preventivo. El motivo reside en la necesidad de educar posturalmente y en la configuración de hábitos activos para afrontar y prevenir posibles anormalidades de hombro favoreciendo un envejecimiento activo (Chacón et al., 2018; Chavarrias, Carlos-Vivas, \& Pérez-Gómez, 2018; Rodríguez, Pérez, Mayoralas, \& Prieto-Flores, 2018; Zurita et al., 2009).

Por tanto, este estudio persigue como principal objetivo determinar la prevalencia de patologías de hombro en adultos mayores de Santiago de Chile(Chile), así como dictaminar la existencia de relación con la práctica de actividad física, el estado de salud percibido y diversos factores sociodemográficos.

\section{Método}

\section{Participantes}

Se realizó un estudio de tipo descriptivo y corte transversal mediante una medición en un único grupo. Participaron en esta investigación un total de 41 adultos de 50 a 65 años $(M=58,17$ años; $D T=$ 
11,75) que acudían regularmente al centro de ejercicio adaptado de la asociación cristiana de jóvenes de Santiago de Chile. La selección de la muestra se realizó por conveniencia, considerando como criterios de inclusión tener una edad superior a 50 años de edad y no padecer ninguna enfermedad de tipo grave. Concretamente, la distribución de los sujetos fue de un 46,3\% ( $=19)$ para los hombres y un 53,7\% ( $n=22)$ para las mujeres.

\section{Instrumentos}

Se emplearon diversos instrumentos validados para evaluar la patología de hombro, estado de salud y actividad físico-deportiva:

Escala de evaluación de hombro «UCLA». Este instrumento se recoge de su versión original planteada por Amstutz, Sew, \& Clarke (1981). Esta escala ha sido empleada a nivel mundial siendo utilizada como el elemento de valoración más reconocido en diversos contextos sanitarios y educativos. Clasifica los hombros mediante un sistema de puntuación, donde establece cinco elementos de valoración en base al dolor, función, flexión activa, fuerza muscular a la flexión activa y satisfacción. Posteriormente se realiza una sumatoria de las puntuaciones obtenidas en las diferentes categorías, la cual permite una clasificación en cuartiles según la evaluación del hombro: excelente (entre 34-35 puntos), bueno (entre 28-33 puntos), regular (entre 21-27 puntos) y malo (entre 0-20 puntos).

Escala Visual del Termómetro de EUROQOL de autovaloración del estado de salud. Este test se valora mediante una escala visual, siendo la segunda parte del EQ-5D, formada por una escala EVA vertical de 20 centímetros, milimetrada, que va desde 0 (peor estado de salud imaginable) a 100 (mejor estado de salud imaginable), como señalan Herdman, Badia, \& Berra (2001). En ella, el individuo debe marcar el punto en la línea vertical que mejor refleje la valoración de su estado de salud global en el día de hoy. El uso de la EVA proporciona una puntuación complementaria al sistema descriptivo de la autoevaluación del estado de salud del individuo.

Cuestionario Ad hoc. Se emplea un cuestionario de elaboración propia con el fin de registrar la edad, el sexo y la realización o no de práctica de actividad física cotidiana considerando al menos tres horas semanales.

\section{Procedimiento}

Se solicitó la colaboración de los centros rehabilitadores de Santiago de Chile para participar en la investigación exponiéndoles de forma breve el objetivo del estudio y solicitando la colaboración de sus pacientes. Asimismo, se adjuntó un modelo de consentimiento informado, junto al protocolo aprobado por el Comité de Ética de la Universidad de Santiago de Chile. La administración de las pruebas se realizó durante los meses de noviembre y diciembre de 2015, siguiendo los pasos del protocolo de aplicación, indicándoles el total anonimato de las respuestas. Los encuestadores estuvieron presentes durante la recogida de los datos para confirmar la correcta realización de las pruebas o test. La recogida se desarrolló sin ningún tipo de anormalidad. Al finalizar los investigadores agradecieron su participación a los encuestados, informándoles de la recepción del informe de los resultados obtenidos al final del estudio en el futuro.

\section{Análisis de los datos}

El análisis estadístico se realizó mediante el Software SPSS 22.0 para Windows. Se consideró un índice de participación del 81,20\%, ya que de la totalidad de sujetos que tomaron parte en este estudio $(n=44)$ tuvieron que eliminarse 3 cuestionarios por estar cumplimentados erróneamente, constituyendo una muestra final de $n=41$. Las técnicas de análisis utilizadas fueron de tipo descriptivo (frecuencias y medias), tablas de contingencia mediante la prueba de chi-cuadrado y una prueba de regresión logística binaria con la variable práctica de actividad física como dependiente. Se incluyeron las variables sexo, estado de salud, patología de hombro y escala de evaluación UCLA en el modelo anterior. La introducción de las variables se hizo manualmente con el criterio de significación e importancia clínica de éstas. Se comprobó la bondad del ajuste del modelo mediante la prueba de Hosmer-Lemeshow.

\section{Resultados}

La tabla 1 determina las frecuencias y medias de los parámetros estudiados. Se muestra que un 53,7\% (n=22) de los participantes tenía tendinopatías del manguito de los rotadores y un 17,1\% ( $\mathrm{n}=7)$ padecía rotura tendinosa. Las patologías restantes (bursitis, hombro congelado y pinzamiento subacromial) estuvieron representadas por un 9,8\% ( $\mathrm{n}=$ 4) de la muestra cada una. En torno a la clasificación del hombro mediante la escala UCLA, un 65,9\% $(\mathrm{n}=27)$ de los participantes se adhirió un estado malo o pobre, mientras que solo un $17,1 \%(n=7)$ denotó un estado de salud excelente o bueno. El estado de salud reveló un valor medio de 68,32 (DT = 16,78).

\begin{tabular}{lcc} 
Tabla 1. & & \\
Descriptivos básicos & & \\
\hline \multicolumn{1}{c}{ Sexo } & Hombre & $46,3 \%(\mathrm{n}=19)$ \\
\cline { 2 - 3 } & Mujer & $53,7 \%(\mathrm{n}=22)$ \\
\hline & Tendinopatías del Manguito de los Rotadores & $53,7 \%(\mathrm{n}=22)$ \\
& Rotura Tendinosa & $17,1 \%(\mathrm{n}=7)$ \\
Patología de Hombro & Bursitis & $9,8 \%(\mathrm{n}=4)$ \\
& Hombro Congelado & $9,8 \%(\mathrm{n}=4)$ \\
& Pinzamiento Subacromial del Manguito & $9,8 \% \mathrm{n}=4)$ \\
\hline & Rotadores & $4,9 \%(\mathrm{n}=2)$ \\
Evaluación UCLA & Excelente & $12,2 \%(\mathrm{n}=5)$ \\
& Bueno & $17,1 \%(\mathrm{n}=7)$ \\
& Regular & $65,9 \%(\mathrm{n}=27)$ \\
\hline Estado de Salud & Malo & $\mathrm{DT}=16,74$ \\
\hline
\end{tabular}

Tabla 2.

Relaciones entre práctica de actividad física, patología de hombro, evaluación UCLA y estado de salud

\begin{tabular}{|c|c|c|c|c|}
\hline \multirow{2}{*}{\multicolumn{2}{|c|}{ Variables }} & \multicolumn{2}{|c|}{ Frecuencias y Medias } & \multirow[b]{2}{*}{$P$} \\
\hline & & Si Practica & No Practica & \\
\hline Sexo & $\begin{array}{l}\text { Hombre } \\
\text { Mujer }\end{array}$ & $\begin{array}{c}39,1 \%(\mathrm{n}=9) \\
60,9 \%(\mathrm{n}=14)\end{array}$ & $\begin{array}{c}55,6 \%(\mathrm{n}=10) \\
44,4 \%(\mathrm{n}=8)\end{array}$ & 0,295 \\
\hline \multirow{5}{*}{$\begin{array}{l}\text { Patología de } \\
\text { Hombro }\end{array}$} & Tendinopatías del MR & $50,0 \%(n=13)$ & $56,5 \%(\mathrm{n}=9)$ & \multirow{5}{*}{0,485} \\
\hline & Rotura Tendinosa & $27,8 \%(n=3)$ & $8,7 \%(\mathrm{n}=5)$ & \\
\hline & Bursitis & $5,6 \%(n=2)$ & $13,0 \%(\mathrm{n}=1)$ & \\
\hline & Hombro Congelado & $5,6 \%(n=2)$ & $13,0 \%(\mathrm{n}=1)$ & \\
\hline & Pinzamiento Subacromial del MR & $11,1 \%(\mathrm{n}=3)$ & $8,7 \%(\mathrm{n}=2)$ & \\
\hline \multirow{4}{*}{ UCLA } & Excelente & $8,7 \%(n=2)$ & $0,0 \%(n=0)$ & \multirow{4}{*}{$0,041^{*}$} \\
\hline & Bueno & $17,4 \%(n=4)$ & $5,6 \%(n=1)$ & \\
\hline & Regular & $26,1 \%(\mathrm{n}=6)$ & $5,6 \%(n=1)$ & \\
\hline & Malo & $47,8 \%(\mathrm{n}=11)$ & $88,9 \%(\mathrm{n}=16)$ & \\
\hline \multicolumn{2}{|r|}{ Estado de Salud } & $\begin{array}{c}M=75,26 \\
D T=14,99\end{array}$ & $\begin{array}{c}M=59,44 \\
D T=14,83\end{array}$ & $0,002^{*}$ \\
\hline
\end{tabular}

La tabla 2 estudia la relación entre la práctica de actividad física, el sexo, la patología de hombro, la evaluación UCLA y el estado de salud. Los resultados arrojan la ausencia de relación entre la patología de hombro y el sexo en cuanto a la práctica regular de actividad física. Sin embargo, se revelan diferencias estadísticamente significativas ( $p d$ ) 0,05 ) en la evaluación del hombro y en los estados de salud. En el primer caso se establece que un $88,9 \%(\mathrm{n}=16)$ de los sujetos que no realizan actividad física obtienen una evaluación mala al aplicarse la escala UCLA, frente al 47,8\% $(n=11)$ de los que si practican. Se puede apreciar un comportamiento similar en el estado de salud donde aquellos sujetos físicamente activos que obtenían un valor medio más elevado ( $M=$ $75,26)$ frente a los que no realizaban actividad física $(M=59,44)$.

En la tabla 3 se presentan los resultados de la regresión logística binaria para predecir el comportamiento de la realización de actividad física en función del sexo, la patología y evaluación de hombro y el estado de salud. Cuando se analiza de forma conjunta se encuentran asociaciones $(p<0,05)$ en el modelo ajustado de regresión lineal. En cuanto a la actividad física con el sexo [OR (ajustada) $=0,086(0,008-$ $0,977)$ ] y el estado de salud [OR (ajustada) $=0,021(0,001-0,311)]$. El modelo se ajusta bien y es significativo, explica entre el 0,383 (R2 de Cox y Snell) y el 0,513 (R2 de Nagelkerke) de la variable dependiente y clasifica correctamente a un $73,2 \%$ de los participantes.

Tabla 3.

\begin{tabular}{|c|c|c|c|c|c|}
\hline & \multirow[b]{2}{*}{ B } & \multirow[b]{2}{*}{ Sig. } & \multirow[b]{2}{*}{ O.R } & \multicolumn{2}{|c|}{ I.C. 95\% } \\
\hline & & & & Inferior & Superior \\
\hline Sexo & $-2,457$ & $0,048^{*}$ & 0,086 & 0,008 & 0,977 \\
\hline Patología de Hombro & $-0,501$ & 0,743 & 0,606 & 0,030 & 12,107 \\
\hline Evaluación UCLA & 1,894 & 0,208 & 6,648 & 0,348 & 126,885 \\
\hline Estado de Salud & $-3,864$ & $0,005^{*}$ & 0,021 & 0,001 & 0,311 \\
\hline Constante & 1,108 & 0,621 & 3,028 & - & - \\
\hline
\end{tabular}




\section{Discusión}

En la presente investigación, similar al realizado en otras poblaciones de adultos, se empleó test UCLA, el cual ha sido ampliamente utilizado para la valoración de anomalía de hombro (Arce, Ferro, Pereira, Arcuri, \& Previgliano, 2012; Arcuri, Abalo, \& Barclay, 2012); siendo actualmente el método no invasivo empleado con mayor asiduidad en el diagnóstico de patologías de tipo articular. Se encontró que más de la mitad de los mayores tenían tendinopatías del manguito de los rotadores, situándose esta población en valores similares a otras zonas americanas y mundiales (Calle \& Hincapie, 2014; Cáceres-Sánchez, Mesa-Mateo, Barrionuevo-Sánchez, García-Benítez, \& Expósito-Triano, 2015).Asimismo, debe considerarse que en la mayor parte de los sujetos analizados se cursaba la disfunción con una evaluación mala del UCLA, datos que confirman lo plasmado por otros estudios, que señalan la insatisfacción y procesos dolorosos de la patología de hombro, motivando puntuaciones medias relativamente bajas en los estados de salud (CáceresSánchez et al., 2015).

La muestra analizada señaló en la mitad de los casos una predisposición a realizar actividad física periódicamente, y entre las tareas que desarrollan los ejercicios de mantenimiento (andar, carrera suave, etc.) fueron los más frecuentes. Se entiende que en estas edades la práctica físico-deportiva no es competitiva, estando más relacionada con fines recreativos, mejorando la salud y la calidad de vida. Esto es debido a que el proceso de envejecimiento varía notoriamente según el estilo de vida, afectando al desempeño de las actividades cotidianas (Berzosa, GómezAngulo, Regodón, \& Santamaría, 2013; Martín, Barriopedro, Martínez del Castillo, Jiménez-Beatty, \& Rivero-Herráiz, 2014).

Los datos obtenidos reportaron que ejercicios de intensidad leve y moderada como son los de mantenimiento son los más arraigados en poblaciones de personas mayores, puesto que son tareas poco invasivas y producen un bienestar físico, mental y social considerable entre sus practicantes. Por tanto, es indudable que la actividad física y su práctica generan beneficios a nivel físico y psicológico (disminución de cansancio percibido, estados de bienestar, etc.), influyendo en la prevención de enfermedades y aportando mejoras en los niveles de autoestima y salud percibida (Dishman et al., 2006). Del mismo modo, Vidarte, Quintero, \& Herazo (2012) señalan que se produce una mejora notoria de la fuerza, estabilidad y resistencia de tipo aeróbico.

No se hallaron diferencias estadísticamente significativas en la relación dada entre la realización de actividad física, el sexo y patología de hombro, por lo que el comportamiento de esta patología es bastante similar en base a estos factores. Donde sí se halló relación fue en aquellos sujetos que no realizaban deporte habitualmente, los cuales mostraron mayor dolor, insatisfacción y un estado de salud más negativo en comparación con el resto que si desarrollaban actividad física, poniendo de manifiesto lo planteado por Kappe et al. (2015) o Zurita (2015) quienes indican que la práctica deportiva produce mejoras y adaptaciones anatómico-fisiológicas que mejoran el estado de salud y promueven un envejecimiento ligado a una menor prevalencia de patologías, por lo que la actividad física es un elemento clave en la prevención de problemas de hombro y en su tratamiento (Verhagen, 2017).

Asimismo, se planteó un modelo de regresión donde se encontró un alto nivel de ajuste, estableciéndose que los sujetos físicamente más inactivos eran las mujeres y los que cursaban con un peor estado de salud. Se constatan los beneficios de la práctica regular de actividades físico-deportivas (Guillén, Bueno, Gutiérrez, \& Guerra, 2018), hecho lógico si partimos del supuesto de la relación entre la actividad física y la calidad de vida, pues los beneficios consecuentes de la implicación activa en el ejercicio se encuentran contrastados (Herrera, Pablos, Chiva-Bartoll, \& Pablos, 2017; Smith, Banting, Eime, O’Sullivan, \& van Uffelen, 2017). Del mismo modo, es posible entender que la relación entre sedentarismo y disminución de la calidad de vida se encuentra asociada, puesto que aquella persona que se encuentre impedida para hacer una actividad tendrá un estado de salud más negativo (Márquez, Hu, Jerome, \& Diener, 2005; McAuley et al., 2006).

Desde el punto de vista de su aplicación práctica este estudio presenta un método de detección simple de alteraciones de hombro y su relación con otros parámetros sociodemográficos, destacando la relevancia de la práctica físico-deportiva como elemento preventivo siempre y cuando esta sea prescrita profesionalmente y atendiendo a las particularidades de cada sujeto. Esto podría ayudar no solo en la disminución del dolor en aquellos adultos mayores que padecen patologías asociadas al hombro, la reeducación de la propia articulación con el fin de evitar la intervención quirúrgica y mejorar el estado de salud percibida y la calidad de vida.

En el desarrollo de esta investigación hemos detectado algunas limitaciones como la de un escaso número de participantes y no haber realizado una extensión del estudio a otras poblaciones chilenas, que nos hubiese dado una mayor perspectiva. Asimismo, se presentan las limitaciones propias de un estudio de tipo transversal, el cual no permite establecer relaciones de causa-efecto, aunque resultan eficaces para conocer el estado de una cuestión.

Finalmente, y como principales conclusiones se concreta que la práctica de actividad física regular es un elemento que potencia el desarrollo de la patología de hombro sin dolor y un mejor estado de salud que aquellos adultos que no realizan ejercicios de mantenimiento y que son más inactivos. Asimismo, los datos obtenidos revelan que ser mujer y tener peor estado de salud se vincula con menores niveles de práctica de actividad física en personas afectas de hombro.

\section{Referencias}

Amstutz, H.C., Sew, A.L., \& Clarke, I.C. (1981). UCLAanatomic total shoulder arthroplasty. Clinical Orthopedic, 155, 7-20.

Arce, G, Ferro, D., Pereira, E., Arcuri, F., \& Previgliano, J.P. (2012). ¿Es la cirugía artroscópica adecuada para las revisiones en inestabilidad de hombro?: selección de pacientes y resultados. Artroscopia de Buenos Aires, 19(1), 43-49.

Arcuri, F., Abalo, E., \& Barclay, F. (2012). Uso de Escores para Evaluación de la Inestabilidad de Hombro. Artroscopia de Buenos Aires, 19(1), 67-72.

Berzosa, J., Gómez-Angulo, J., Regodón, C., \& Santamaría, L. (2013). ¿Te cuidas? Hábitos de vida de las mujeres de la Comunidad de Madrid. Madrid: Comunidad de Madrid, Consejería de Asuntos Sociales.

Breckenridge, J. D., \& McAuley, J. H. (2011). Shoulder pain and disability index (SPADI). Journal of Physiotherapy, 57(3), 197202.

Cáceres-Sánchez, L., Mesa-Mateo, A., Barrionuevo-Sánchez, F.J., García-Benítez, B. \& Expósito-Triano, S. (2015). Artroplastia total invertida de hombro. Evaluación de resultados clínicos y complicaciones tras una serie de 52 casos. Revista Española de Cirugía Ortopédica y Traumatología, 59(6), 439-446.

Calle, Y. \& Hincapie S. (2014). Síndrome de pinzamiento del hombro: una revisión de tema. Revista CES Movimiento y Salud, 2(1), 3244.

Calvo,A.,Arce, G., \& Calvo, E. (2009). La clasificación MIQ: buscando el consenso para clasificar el hombro inestable. Cuadernos de Artroscopia, 16, 22-33.

Chacón, R., Chacón, F., Zurita, F., Cachón, J., Zagalaz, M. L., Chinchilla, J. J. (2018). Characterization of motivation and type of physicalsport practice in adults through COMPASS profiles. Journal of Human Sport and Exercise, 13(1), 161-173.

Chavarrias, M., Carlos-Vivas, J., \& Pérez-Gómez. (2018). Beneficios para la salud de Zumba: una revisión sistemática. Journal of Sport and Health Research, 10(3), 327-338.

Crotin, R., Bhan, S., \& Ramsey, D. (2015). An inferential investigation into how stride length influences temporal parameters within the baseball pitching delivery. Human Movement Science, 41, 127135.

Dishman, R.K., Hales, D.P., Pfeiffer, K.A., Felton, G., Saunders, R., Ward, D.S., \& Pate, R.R. (2006). Physical self-concept and selfesteem mediate cross-sectional relations of physical activity and 
sport participation with depression symptoms among adolescent girls. Health Psychology, 25(3), 396-407.

Furness, J., Schram, B., Cottman-Fields, T., Solia, B., \& Secomb, J. (2018). Profiling Shoulder Strength in Competitive Surfers. Sports, 6(2), 52.

Guillén, L., Bueno, E., Gutiérrez, M., \& Guerra, J. (2018). Programa de actividad física y su incidencia en la depresión y bienestar subjetivo de adultos mayores. Retos: Nuevas tendencias en Educación Física, Deporte y Recreación, (33), 14-19.

Hegedus, E. J., Cook, C., Lewis, J., Wright, A., \& Park, J. Y. (2015). Combining orthopedic special tests to improve diagnosis of shoulder pathology. Physical Therapy in Sport, 16(2), 87-92.

Herdman, M., Badia, X., \& Berra S. (2001). El EuroQol-5D: una alternativa sencilla para la medición de la calidad de vida relacionada con la salud en atención primaria. Atención Primaria, 28(6), 425-429.

Herrera, E., Pablos, A., Chiva-Bartoll, O., \& Pablos, C. (2017). Efectos de la Actividad Física en la Salud Percibida y Condición Física de los Adultos Mayores. Journal of Sport and Health Research, 9(1), 27-40.

Hinds, R., Garner, M., Tran, W., Lazaro, L., Dines, J., \& Lorich, D. (2015). Geriatric proximal humeral fracture patients show similar clinical outcomes to non-geriatric patients after osteosynthesis with endosteal fibular strut allograft augmentation. Journal of Shoulder and Elbow Surgery, 24, 889-896.

Kappe, T., Knappe, K., Elsharkawi, M., Reichel, H., \& Cakir, B. (2015). Predictive value of preoperative clinical examination for subacromial decompression in impingement syndrome. Knee Surgery, Sports Traumatology, Arthroscopy, 23, 443-448.

Jimenez-Olmedo, J. M., Penichet-Tomás,A., Pueo, B., Chinchilla_Mira, J. J., \& Pérez-Turpín, J. A. (2018). Patrón lesivo en Campeonato de España universitario de vóley playa. Revista Internacional de Medicina y Ciencias de la Actividad Física y del Deporte, 18(70), 331-340.

Karel, Y. H., Scholten-Peeters, G. G, Thoomes-de Graaf, M., Duijn, E., van Broekhoven, J. B., Koes, B. W., \& Verhagen, A. P. (2017). Physiotherapy for patients with shoulder pain in primary care: a descriptive study of diagnostic-and therapeutic management. Physiotherapy, 103(4), 369-378.

Márquez, D.X., Hu, L., Jerome, G.J., \& Diener, E. (2005). Physical Activity Enhances Long-Term Quality of Life in Older Adults: efficacy, esteem and affective influences. Annals of Behavioral Medicine, 31(1), 99-103.

Martín, M., Barriopedro, M.I., Martínez del Castillo, J., JiménezBeatty, J.E., \& Rivero-Herráiz,A. (2014). Diferencias de género en los hábitos de actividad física de la población adulta en la Comunidad de Madrid. Revista Internacional de Ciencias del Deporte, 38(10), 319-35.
McAuley, E., Konopack, J.F., Motl, R.W., Morris, K.S., Doerksen, S.E., \& Rosengren, K.R. (2006). Physical Activity and Quality of Life in Older Adults: Influence of Health Status and Self-Efficacy. Annals of Behavioral Medicine, 31(1), 99-103.

Parra, P. G., Rojas, M. A., Bravo, B. J., Oria, M. G, Muñoz, M. L., Álvarez,J. G., \& Luis, P. C. (2016). Correlación entre la exploración física y los hallazgos intraoperatorios de patología de hombro tratada mediante artroscopia. Análisis estadístico de 150 pacientes. Revista Española de Cirugía Ortopédica y Traumatología, 60(5), 306-314.

Patel, K. (2009). Corrective Exercise. A practical approach. Londres: Hodder Arnold.

Peixoto, G., Fiquetti, P., Polisello, N., Barbosa, G., Ejnisman, B.,

\& Cohen, M. (2013). Glenohumeral range of motion in handball players with and without throwing-related shoulder pain. Journal Shoulder Elbow Surgery, 22, 602-607.

Razmjou, D., Boljanovic, D., Lincoln, S., Geddes, C., Macritchie, I., Virdo-Cristello, C., \& Richards, R. (2015). Examining outcome of early physician specialist assessment in injured workers with shoulder complaints. BMC Musculoskeletal Disorders, 16(1), 32.

Rodríguez, V. R., Pérez, F. R., Mayoralas, G. F., \& Prieto-Flores, M. E. (2018). ¿Cómo interpretan el envejecimiento activo las personas mayores en España?: Evidencias desde una perspectiva no profesional. Aula Abierta, 47(1), 67-78.

Sharma, S., Bærheim,A., \& Kvåle, A. (2015). Passive range of motion in patients with adhesive shoulder capsulitis, an intertester reliability study over eight weeks. BMC Musculoskeletal Disorders, 16(1), 37.

Smith, G. L., Banting, L., Eime, R., O’Sullivan, G., \& van Uffelen, J. G. (2017). The association between social support and physical activity in older adults: a systematic review. International Journal of Behavioral Nutrition and Physical Activity, 14(1), 56.

Vidarte, J.A., Quintero, M.V., \& Herazo, Y.(2012). Efectos del ejercicio físico en la condición física funcional y la estabilidad en adultos mayores. Hacía la promoción de la salud, 17(2), 79-90.

Waterman, B., Cameron, K., Hsiao, M., Langston, J., Clark, N., \& Owens, B. (2015). Trends in the diagnosis of SLAP lesions in the US military. Knee Surgery, Sports Traumatololgy, Arthroscopy, 23, 1453-1459.

Zurita, F. (2015). Influencia de los factores psicológicos sobre las lesiones deportivas en deportes de equipo. Tesis Doctoral: Universidad de Jaén.

Zurita, F., Fernández, R., Cepero, M., Zagalaz, M.L., Valverde, M., \& Ramírez, P. (2009). The relationship between pain and physical activity in older adults that begin a program of physical activity. Journal of Human Sport and Exercise, 4(3), 284-297.



